\title{
A síndrome de Cri Du Chat em adolescentes
}

\section{The Cri du Chat syndrome in adolescents}

Erlane Marques Ribeiro ${ }^{1,2}$ (1) , Estela Mares Santos Salmito Matos ${ }^{3}$ (D) , Leonardo Siqueira Albuquerque ${ }^{3}$ (D), Luzia Julia Porto Carneiro $^{4}\left(\mathbb{0}\right.$, Regina Coeli de Carvalho Porto Carneiro ${ }^{5}(\mathbb{0}$

1. Docente do Curso de Medicina do Centro Universitário Christus (UNICHRISTUS), Fortaleza, CE, Brasil. 2. Hospital Infantil Albert Sabin (HIAS), Fortaleza, CE, Brasil. 3. Discente do Curso de Medicina do Centro Universitário Christus (UNICHRISTUS), Fortaleza, CE, Brasil. 4. Discente do Curso de Medicina do Centro Universitário INTA (UNINTA), Sobral, CE, Brasil. 5. Docente do Curso de Medicina do Centro Universitário INTA (UNINTA), Sobral, CE, Brasil.

\section{Resumo}

Introdução: A síndrome do miado do gato ou síndrome 5p é uma doença congênita rara causada por uma anormalidade cromossômica. Relato do caso: Apresentamos o caso de uma paciente de 13 anos com características dismórficas leves. 0 retardo mental era grave, com comportamento mal adaptativo e hiperatividade. 0 diagnóstico citogenético da anormalidade cromossômica 46 XX del 5pter-->p13 foi estabelecido. Conclusão: 0 diagnóstico precoce dessa doença é necessário para a qualidade de vida dos pacientes, mas esse diagnóstico é difícil de ser realizado em pacientes que foram vistos, pela primeira vez, em idade mais avançada.

Palavras-chave: Aneuploidia. Aconselhamento Genético. Síndrome de Cri-du-Chat.

\section{Abstract}

Introduction: Cat cry syndrome or 5p-syndrome is a rare congenital disease caused by a chromosomal abnormality. Case report: The case of a thirteenyear-old female patient with mild dysmorphic features is presented. Mental retardation is severe, with maladaptive behavior and hyperactivity. The cytogenetic diagnosis of chromosomal abnormality karyotype $46 \mathrm{XX}$ del 5 pterp13 has been established. Conclusions: We concluded that early diagnosis of this disease is necessary for patients' quality of life, but this diagnosis is difficult to make in patients who were first seen at an older age.

Keywords: Aneuploidy. Genetic counseling. Cri-du-Chat syndrome.

\section{INTRODUÇÃO}

A síndrome de Cri du Chat (SCC), descrita, pela primeira vez, por Jerome Lejeune, em 19631, é uma síndrome cromossômica rara, com incidência de 1:15.000-50.000 nascidos vivos ${ }^{2}$. As características clínicas no período neonatal são microcefalia, dismorfismo facial e choro em miado de gato, que deu o nome à síndrome ${ }^{1-3}$.

Por ser uma doença genética, é importante o diagnóstico etiológico, pois, em alguns casos, a família pode estar em risco de uma maior recorrência da doença. A realização do aconselhamento genético faz parte das boas práticas de atendimento clínico, colaborando para adaptação da família à condição clínica da paciente em questão ${ }^{3}$

Nesse contexto, apresentamos o caso de uma adolescente com SCC e a dificuldade em realizar o diagnóstico, após o período neonatal, devido à semelhança do quadro clínico com outras doenças neurológicas.

\section{RELATO DO CASO}

O presente relato de caso foi aprovado pelo Comitê de
Ética em Pesquisa do Hospital Infantil Albert Sabin CAAE 78568717.0.0000.5042. Trata-se de uma paciente de 13 anos, sexo feminino, segunda filha de pais saudáveis e não consanguíneos, cuja mãe tem 27anos e o pai, 44 anos na ocasião do nascimento. Trata-se de uma segunda gestação planejada, sem intercorrências. Nega abortos. Foi diagnosticado pé torto congênito bilateral ao ultrassom no pré-natal. $O$ parto foi normal a termo com 38 semanas de gestação. Não necessitou de manobras de reanimação. Peso de nascimento: 2680g, comprimento: $45 \mathrm{~cm}$ e perímetro cefálico: $34 \mathrm{~cm}$. Sem história de hipóxia neonatal.

No exame físico, constataram-se hipotonia, microcefalia, dismorfismos faciais e pé torto congênito bilateral (figura 1). $\mathrm{Na}$ evolução, houve importante atraso no desenvolvimento neurológico, apesar da realização da estimulação essencial e terapias de reabilitação. Quanto aos marcos de desenvolvimento, sustentou a cabeça aos 7 meses, sentou-se aos 2 anos e 6 meses, nunca adquiriu a marcha nem apresentou linguagem expressiva. Atualmente, a paciente não compreende ordens verbais simples, interage, pobremente, com o meio; porém reconhece familiares, manifestando satisfação ao 
vê-los. O exame físico neurológico apresentou movimentos estereotipados, espasticidade e sinais de liberação piramidal. (Figura 2). Quanto aos exames complementares, realizou tomografia de crânio que evidenciou atrofia cortical moderada e o exame de raios $X$ de coluna total mostrou escoliose importante. $O$ cariótipo foi $46 \mathrm{XX}$ del 5 pter-->p13. A paciente era atendida na APAE do município e realizou aconselhamento genético. Foi solicitado o cariótipo dos pais.

Figura 1. Paciente com S. Cri du Chat com 13 anos (close de face)

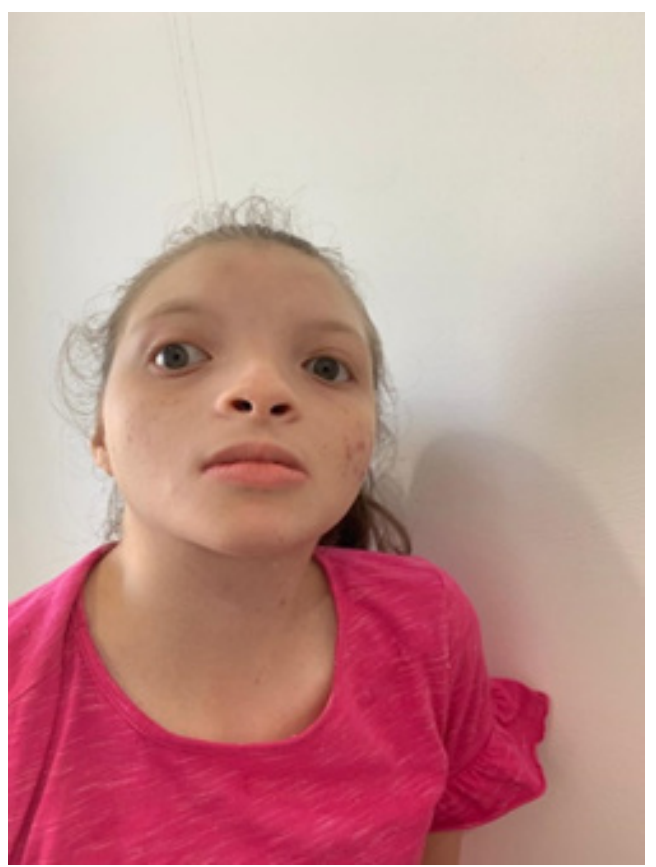

Figura 2: Paciente de 13 anos com S. Cri du Chat (corpo inteiro)

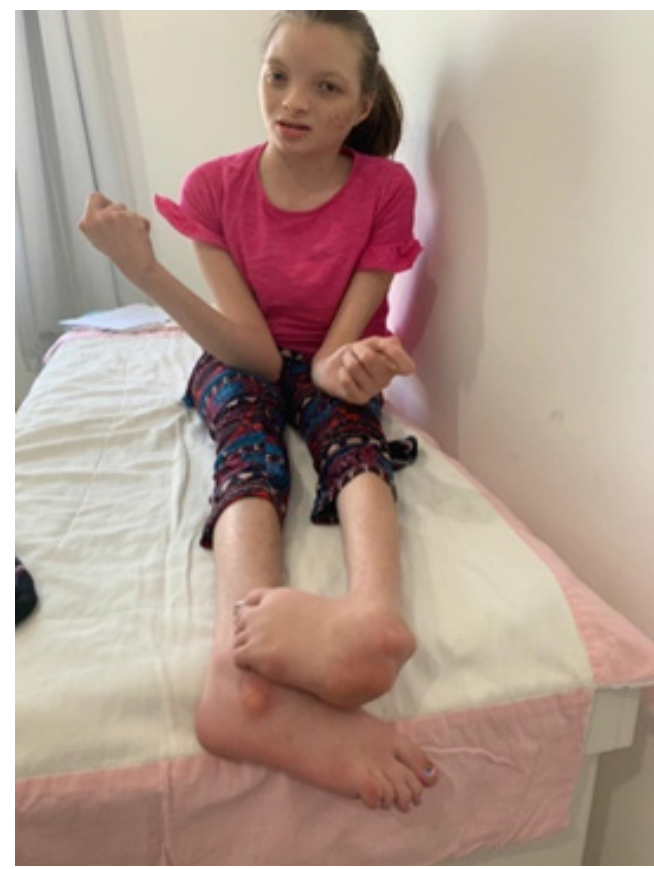

\section{DISCUSSÃO}

O quadro clínico da SCC é mais fácil de se reconhecer no período neonatal, em que há microcefalia, déficit de crescimento, hipotonia, dificuldade para se alimentar, dismorfismos faciais, caracterizados por face arredondada, hipertelorismo, fissura palpebral inclinada para baixo, epicanto, orelhas de baixa implantação, ponte nasal alargada, micrognatia. O "choro miado de gato", achado característico nesse período, mas não patognomônicoda SCC; é definido como um choro de alto volume, monocromático e que desaparece com a idade, tornando o diagnóstico da síndrome mais difícil ${ }^{2-5}$.

Nesses casos em que o diagnóstico é mais tardio, os sintomas são mais inespecíficos e mais brandos, podendo confundilos com o diagnóstico etiológico e com outras doenças que apresentam alterações neurológicas ${ }^{6}$.

Com a idade, a face se torna alongada, a fissura palpebral horizontalizada, aparecem macrostomia e escoliose, como no caso que apresentamos.Os cabelos podem- se tornar brancos precocemente e é comum o aparecimento de distúrbio de comportamento, caracterizado por comportamento destrutivo, automutilação e agressividade. Essas alterações de comportamento são causas de consulta ao psiquiatra, que pode estar manejando os sintomas sem reconhecer que se trata de um paciente com SCC2, ${ }^{7-9}$.

A SCC ocorre por deleção de parte ou totalidade do braço curto do cromossomo 5; então, quando existe uma suspeita clínica da SCC, deve-se solicitar o exame do cariótipo com banda G no sangue periférico, que detecta perda ou ganho de grandes partes ou do todo de algum cromossomo ${ }^{2}$. Esse teste é o de menor custo no rol dos exames da citogenética e foi o suficiente para o diagnóstico da paciente que apresentamos.

Nos casos em que o cariótipo é normal e a suspeita clínica persiste, deve-se realizar o FISH (hibridização fluorescente in situ) 10para SCC ou CGH (hibridização genômica comparativa), pois a alteração citogenética pode ser pequena, e a resolução do cariótipo não seria suficiente para a detectá-la $a^{4,9,11}$.

Em relação ao aconselhamento genético, apesar de a maioria dos casos de SCC ser decorrente de mutação nova (80\%) com risco de recorrência desprezível, devemos solicitar cariótipo com banda $\mathrm{G}$ no sangue periférico para os pais que têm filho com SCC, pois, em $10 \%$ desses casos, o risco de recorrência pode ser maior devido a um rearranjo cromossômico, balanceado no material genético de um dos pais, podendo ser inversão ( $10 \%$ dos casos) e translocação balanceada ( $90 \%$ dos casos) $)^{2,5}$. Nos casos de haver idade avançada dos pais ou impedimento de uma nova gestação, o cariótipo dos pais ainda se aplica se houver irmãos do afetado, pois algum irmão poderá herdar a alteração cromossômica balanceada de um dos pais e terá maior risco para ter um filho com SCC.

As alterações clínicas na SCC dependem do tamanho, da posição da deleção e mudam com o passar do tempo4. Como 
a paciente apresentada teve deleção de quase totalidade do braço curto do cromossomo 5 (figura 3) com perda de genes contíguos, o fenótipo era grave, com deficiência intelectual importante, ausência da fala, movimentos estereotipados e espasticidade nos quatro membros. Esse quadro clínico ainda se confunde com outras doenças, como autismo, síndrome de Rett, síndrome de Smith-Magenis, síndrome de Angelman e paralisia cerebral decorrente de sequela de hipóxia neonatal.

Figura 3. Tamanho da deleção da paciente cujo cariótipo foi 46 XX del 5 pter-->p13

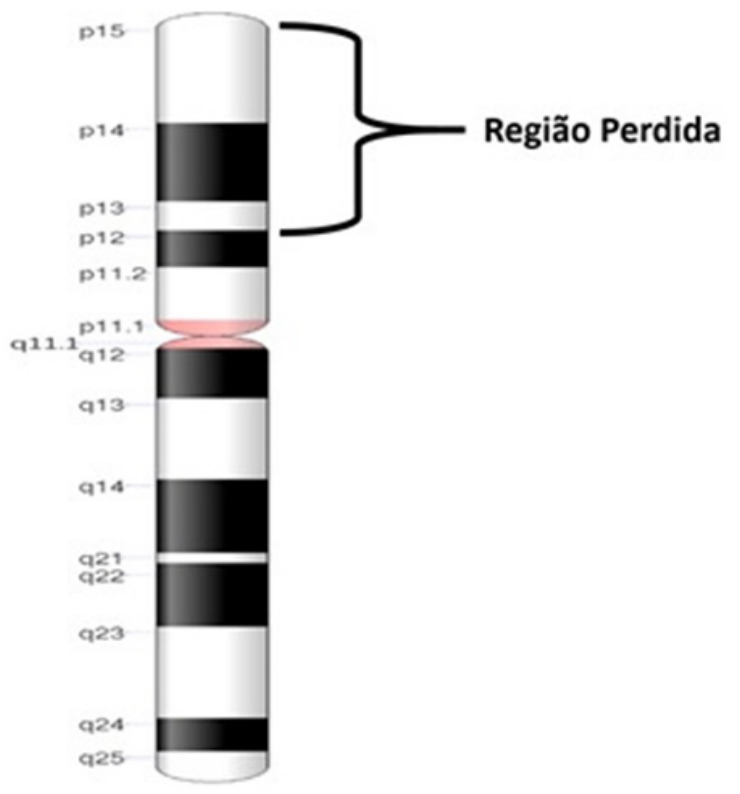

As alterações cardíacas na SCC têm uma prevalência de 15 a $20 \% 2,4$ e não foram observadas na paciente que descrevemos, concorrendo para uma maior sobrevida.

Realizar diagnóstico de SCC na fase adulta é mais difícil, porque os dismorfismos faciais são mais brandos; porém, mesmo nessa idade, o diagnóstico de SCC é importante por permitir o adequado aconselhamento genético, ajudando as famílias a se adaptarem à doença, revelando o risco de recorrência na família.

Os afetados pela SCC podem ter melhorias significativas na qualidade de vida se forem introduzidos, precocemente, às práticas, como fisioterapia e fonoaudiologia2, porém o número de programas de reabilitação diminui conforme os pacientes ficam mais velhos, gerando uma perda progressiva das habilidades adquiridas. Concomitante a esse cenário, os pais envelhecem e perdem, aos poucos, a resistência emocional e física, dificultando a vida social dos afetados.

Concluindo, os sinais dismórficos da SCC devem ser reconhecidos em idade precoce na vida, pois a mudança do fenótipo, com a idade, dificulta a suspeita diagnóstica devido às características clínicas que se tornam mais brandas, e o quadro clínico se confunde com outras doenças associadas a alterações neurológicas. A paciente apresentada teve confirmação da SCC por meio do cariótipo e deve manter a terapia de reabilitação, visando à melhor qualidade de vida.

\section{REFERÊNCIAS}

1. Santana HE, Tamayo CV, Bruzón HM. Síndrome de $5 p$ menos. Presentación de un caso. Medisur [Internet]. 2014 [acesso 201908 19]; 12(4): 650-54. Disponível em: http://scielo.sld.cu/scielo.php?script=sci_arttext\&pid=S1727897X2014000400009\&Ing=es.

2. Maria EL, Alice S, Cesare D, Michela M, Simona C, Marianna S, et al. Children and adults affected by Cridu Chat syndrome: Care's recommendations. Pediatr Rep. 2019 Fev; 11(1): 1-5. doi: 10.4081/pr.2019.7839.

3. Mainard PC. Cri du Chat syndrome. Orphanet J Rare Dis. 2006 Set; 1 (3): 1-9.

4. Mainard PC, Porfino C, Cali A, Coucourd G, Pastores G, Cavani S et al. Clinical and molecular characterisation of 80 patients with $5 p$ deletion: genotypephenotype correlation. J Med Genet. 2001 Mar; 38(3): 151-8. doi: 10.1136/ jmg.38.3.151.

5. Honjo RS, Mello CB, Pimenta LSE, Nunes-Vaca EC, Beneditto LM, Khoury RBF, Befi-Lopes DM, Kim CA. Cri-du-Chat syndrome: characteristics of 33 Brazilian patients. J Intellect Disabil Res. 2018 Jun; 62(6): 467-73. doi: 10.1111/jir.12476.

6. Van Buggenhout GJCM, Pijkels E, Holvoet M, Schaap C, Hamel BCJ, FrynsJP. Cri Du Chat changing phenotype in older patients. Am J Med Genet 2000 Jan; 90(3): 203-15. doi: 10.1002/(sici)1096-8628(20000131)90:3<203::aidajmg5>3.0.co;2-a.
7. Corrêa T, Feltes BC, Riegel M. Integrated analysis of the critical region $5 p 15.3-$ p15.2 associated with cri-du-chat syndrome. Genet. Mol. Biol. [Internet]. 2019 [acesso 2019 Dez 26]; 42(1 Suppl 1): 186-196. Disponível em: http://www.scielo. $\mathrm{br} / \mathrm{scielo}$.php?script=sci_arttext\&pid=S1415-47572019000200186\&Ing=en . EpubApr11, 2019. http://dx.doi.org/10.1590/1678-4685-gmb-2018-0173.

8. Espirito Santo LD, Morena LMA, Riegel M. Cri-du-Chat syndromes: clinical profile and chromosomal microarray analysis in six patients. Biomed Res Int. 2016; 2016: 1-9. doi: 10.1155/2016/5467083.

9. Saldarriaga W, Collazos-Saa L, Ramírez-Cheyne J. Cri-du-chat syndrome diagnosed in a 21-year-old woman by means of comparative genomic hybridization. rev.fac.med. [Internet]. 2017 Set [acesso 2019 Dez 26]; 65(3): 525-529. Disponível em: http://www.scielo.org.co/scielo.php?script=sci arttext\&pid=S0120-00112017000300525\&Ing=en. http://dx.doi.org/10.15446/ revfacmed.v65n3.57414.

10. Ferreiros $M R$,López $M L$,Alonso CC. Accidental finding of a cri du chat syndrome in an adult patient by means of array-CGH. Rev Neurol. 2014 Jul; 16; 59(2): 71-6.

11. Mosca AL,Callier P,Leheup B,Marle N,Jalloul M,Coffinet L, et al. Fortuitous FISH diagnosis of an interstitial microdeletion (5)(q31.1q31.2) in a girl suspected to present acri-du-chatsyndrome. Am J Med Genet A. 2007 Jun; 15;143(12):1342-7. doi: 10.1002/ajmg.a.31742. 